\title{
Bikondyläre Knieendoprothetik Indikation, Technik, Ergebnisse und neue Entwicklungen
}

\author{
$\square$ Christian Lüring, Holger Bäthis, Joachim Grifka
}

\section{Zusammenfassung}

Innerhalb der vergangenen 15 Jahre hat sich die Knieendoprothetik zu einer Standardoperation entwickelt mit guten bis sehr guten Ergebnissen in über 85 Prozent der Fälle. Den Zahlen der Bundesgeschäftsstelle für Qualitätssicherung zufolge wurden in Deutschland in 2005 über 130000 Knietotalendoprothesen implantiert. Trotz der hohen Standardisierung erfordert die Implantation einer Knietotalendoprothese einen erfahrenen Operateur und bietet einige Fehlermöglichkeiten, die zu einer verfrühten Lockerung der Prothese und damit einer weiteren Operation für den $\mathrm{Pa}$ tienten führen können. Die vorliegende Arbeit soll die Indikation, die Technik, die Ergebnisse und die neuesten Entwicklungen auf diesem Gebiet darstellen.
Bicondylar Total Knee Replacement - Indication, Technique, Results and Perspectives

Total knee replacement has developed to a standard operative procedure during the last 15 years. The current literature shows promising results in over 85 percent of the cases. According to the German "Bundesgeschäftsstelle für Qualitätssicherung” over 130,000 total knee replacements were performed in Germany in 2005. Although the procedure has been optimized and standardized, the implantation of a bicondylar knee prosthesis is still a highly demanding procedure. Inaccuracies and errors can result in early loosening of the prosthesis and necessitate another operation for our patient. The aim of this study is to present the indication for implantation, the technique, the results and the latest developments in total knee replacement.

\section{Einleitung}

Die Entwicklungen der letzten Jahre haben dazu beigetragen, dass sich die Knieendoprothetik als Behandlungsstandard der fortgeschrittenen Gonarthrose etablieren konnte. Aus großen Erhebungen wie zum Beispiel dem schwedischen Knieprothesenregister kann bei etwa $80 \%$ der behandelten Patienten eine hohe subjektive Zufriedenheit erwartet werden [46]. Die Standzeit der Prothesen wird zum aktuellen Zeitpunkt je nach Studie mit $85 \%$ nach zehn Jahren angegeben. Dabei stellt das Hauptproblem die aseptische Prothesenlockerung mit unterschiedlichen Ursachen dar: Einerseits aufgrund des Polyethylenabriebs $[16,20,26]$, andererseits aufgrund ver-

OP-JOURNAL 2006; 22: 184-189

(C) Georg Thieme Verlag KG Stuttgart · New York bliebener Gelenkinstabilität [46]. Darüber hinaus ist ein postoperatives Malalignment von mehr als drei Grad Achsabweichung im Varus- oder Valgussinne in der Frontalebene ebenfalls ein entscheidender Faktor. Während in den vergangenen Jahrzehnten vor allem die Weiterentwicklungen des Prothesendesigns und der Materialien im Mittelpunkt standen, rückte in den letzten Jahren zunehmend die Optimierung der Operationstechnik in den Vordergrund des wissenschaftlichen Interesses. In verschiedenen Langzeitstudien zum Kniegelenkersatz konnte eine exakte Ausrichtung von Prothesenkomponenten als bedeutsam für die Langzeitstabilität ermittelt werden [27, 43, 45]. Vor diesem Hintergrund wurde seit 1998 parallel zur Verbesserung der konventionellen mechanischen Ausrichthilfen mit der Entwicklung der bereits in der Neurochirurgie und Wir- belsäulenchirurgie etablierten Navigationssysteme auf die Anforderungen der Endoprothetik begonnen [47], um der besonderen Bedeutung der Achsausrichtung gerecht zu werden. Die vorliegende Arbeit soll die Indikationen, die Standardoperationstechnik, die Ergebnisse und die aktuellen Entwicklungen darstellen.

\section{Epidemiologie der Gonarthrose}

Bei der Gonarthrose handelt es sich um eine degenerative und fortschreitende Erkrankung des Kniegelenks, die mit einer Zerstörung des Gelenkknorpels und im späteren Stadium des gelenknahen Knochens, der Synovia und des periartikulären Kapsel-Band-Apparates einhergeht.

Die Angaben des Statistischen Bundesamts verdeutlichen, dass die behandlungsbedürftige Gonarthrose ihren Gipfel in der sechsten bis siebten Lebensdekade besitzt. Hier leiden 26\% der Frauen und $21 \%$ der Männer unter einer behandlungsbedürftigen Arthrose des Kniegelenks.

Indikation zur Knieprothese besteht bei Nacht-, Ruhe- und Belastungsschmerz sowie Reduktion der Gehstrecke.

\section{Indikation}

Die Indikation zur Implantation einer Knietotalendoprothese besteht bei fortgeschrittenen arthrotischen Veränderungen des Kniegelenks. Je nach klinischem und radiologischem Befundbild sollten mit dem Patienten mögliche Alternativen (Umstellungsosteotomie bei jungen Patienten und noch ausreichender Knorpeldecke), arthroskopische Revisionen (Zeitgewinn?), unilateraler Gelenkersatz (isolierte mediale oder laterale Arthrose ohne Patellabefall) kritisch diskutiert werden. Erst bei Ausschöpfen der konservativen Maßnahmen (Physiotherapie, regelmäßige Schmerzmedikation, physikalische Anwendungen), Nacht- 
schmerz, Ruheschmerz, Reduktion der Gehstrecke auf wenige 100 Meter ist die Indikation zur Endoprothesenimplantation gerechtfertigt.

\section{Operative Technik}

\section{Zugang}

Trotz des hoch standardisierten Verfahrens der Implantationstechnik bestehen speziell zu den unterschiedlichen Zugängen aus anatomischer Sicht noch differierende Meinungen [16, 34, 44].

Die jeweiligen Vor- und Nachteile der Zugangswege werden weiterhin kontrovers und bisher noch nicht abschließend diskutiert. Von einigen Autoren wird der weitverbreitete medial-parapatellare $\mathrm{Zu}$ gang wegen der Beeinflussung des Streckapparates als nachteilig angesehen $[9,15$, 18]. Einige Studien zeigen die Unterlegenheit gegenüber dem Sub- und Midvastuszugang in der Frührehabilitation [9, 17, 30, 35, 41]. Diese Arbeiten haben zu einer weiteren Verbreitung dieser Zugangswege beigetragen. Da Subvatusund Midvastuszugang sich an anatomischen Strukturen orientieren $(15,16$, 17), erhalten sie weitestgehend die Integrität des proximalen Streckapparates. Aufgrund der weitesten Verbreitung wird im Weiteren der medial-parapatellare Zugangsweg zum Gelenk beschrieben (Abb.1).

Der medial-parapatellare Zugang ist am weitesten verbreitet.

Nach meist medianer Hautinzision wird anschließend der Zugang zum Gelenk angelegt. Hier ist bei der medial-parapatellaren Inzision darauf $\mathrm{zu}$ achten, dass die Quadrizepssehne im Verhältnis 1/3 zu $2 / 3$ zum M. vastus medialis inzidiert wird und bis ca. $5 \mathrm{~cm}$ oberhalb der Patella eingekerbt wird. Nach distal ist eine Verletzung der Patellarsehne unbedingt zu vermeiden. An der Patella sollte ein Kapselstreifen von ca. $1 \mathrm{~cm}$ Breite belassen werden, der einen sicheren Kapselverschluss zulässt. Im weiteren Verlauf wird die Gelenkkapsel über eine Breite von ca. $1-2 \mathrm{~cm}$ vom medialen Tibiaplateau knochennah abpräpariert, um eine optimale Übersicht über das Gelenk zu erhalten. Das Ligamentum intermeniscale anterius wird inzidiert, das vordere Kreuzband wird exzidiert und der Hoffasche Fettkörper wird soweit möglich belassen, um die propriozeptiven Eigenschaften nicht zu gefährden. Je nach Vorgehensweise wird nun die Synovialitis im oberen Recessus entfernt, um eine optimale Sicht auf die ventrale Kortikalis des Femur zu erhalten. Je nach Versorgung der Patella kann diese anschließend evertiert werden und einer dachziegelartigen Resektionsarthroplastik (Abb.2) mit zirkulärer Denervierung oder einer Osteophytenabtragung mit Denervierung zugeführt werden. Soll die Patella ersetzt werden, bietet sich dies nach der Implantation der Femur- und Tibiakom-

\section{Knochenzurichtung}

Prinzipiell bestehen nun zwei Möglichkeiten der Vorgehensweise: die Femurfirst- oder die Tibia-first-Variante. Dies hängt zum einen vom verwendeten Implantat ab und zum anderen von der Philosophie des Weichteilmanagements. In der Regel und beim „Standardknie“ ohne wesentliche Achsdeformitäten wird der Operateur die Femur-first-Variante wählen.

\section{Femur}

Hier wird nun leicht medio-ventral der interkondylären Notch der Eintrittspunkt für die intramedulläre Führung aufgemeißelt und der intramedulläre Stab entsprechend eingebracht. Die möglichen Achsfehler durch ungünstige Wahl des Eintrittspunktes sind hinreichend beschrieben [13, 44]. Anschließend kann der distale Femurschnitt geführt werden. ponente an.

Hier ist insbesondere bei stark sklerosiertem Knochen zu überprüfen, ob die Resektion mit der Planung übereinstimmt, da ansonsten Sägeschnittfehler zu Achsabweichungen führen können [39, 42]. Die Größenmesslehre wird im Weiteren aufgebracht und einerseits die Prothesengröße bestimmt und andererseits die Rotation der Femurkomponente festgelegt (Abb.3). Ist die Rotation markiert, kann der sogenannte „Vier-ineins"-Schnittblock aufgebracht werden und die Femurresektion wird vollendet. Es bietet sich anschließend an, die Reste der Menisken vorsichtig (speziell medial) zu entfernen und den „fünften Femurschnitt" durchzuführen: das Abschlagen von Osteophyten an den dorsalen Femurkondylen (Abb.4), die eine vollständige Streckung durch Spannung der Gelenkkapsel verhindern.

\section{Wichtig ist der „S-Femurschnitt“.}

\section{Tibia}

Ist dies vollbracht wendet sich der Operateur der Tibia zu und wird in der Regel extramedullär den Schnittblock ausrichten. Hierbei ist besonders auf die VarusValgus-Einstellung zu achten, sodass der Tibiaschnitt senkrecht zur Tibiaachse vorgenommen werden kann. Es bietet sich aus Sicht der Autoren an, eher die weniger defekte Seite (bei der Varusgon-

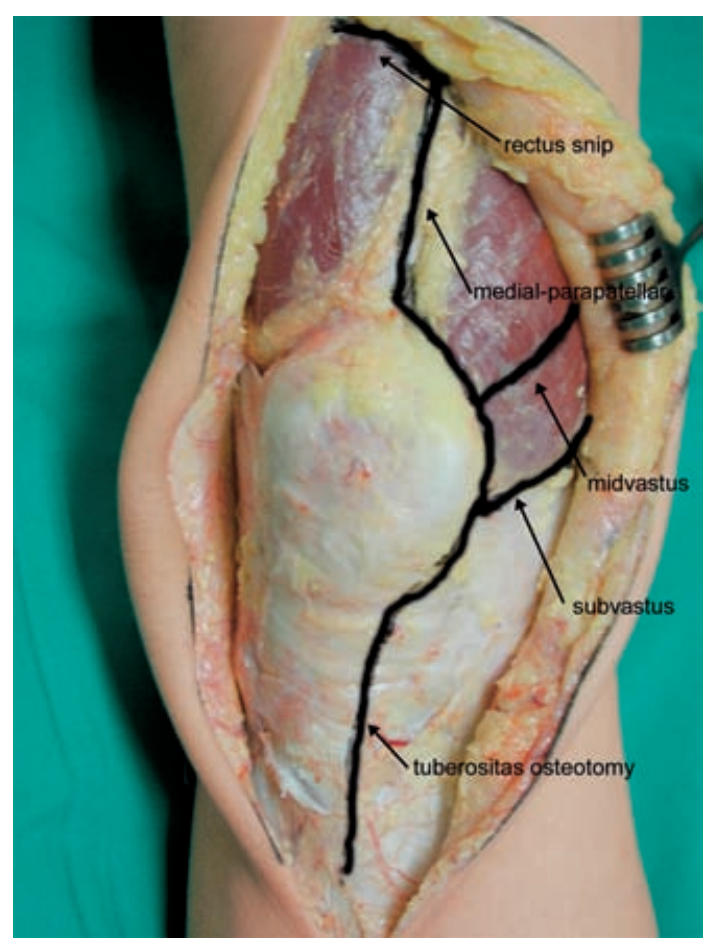

Abb. 1 Anatomische Zugangswege am Präparat. 


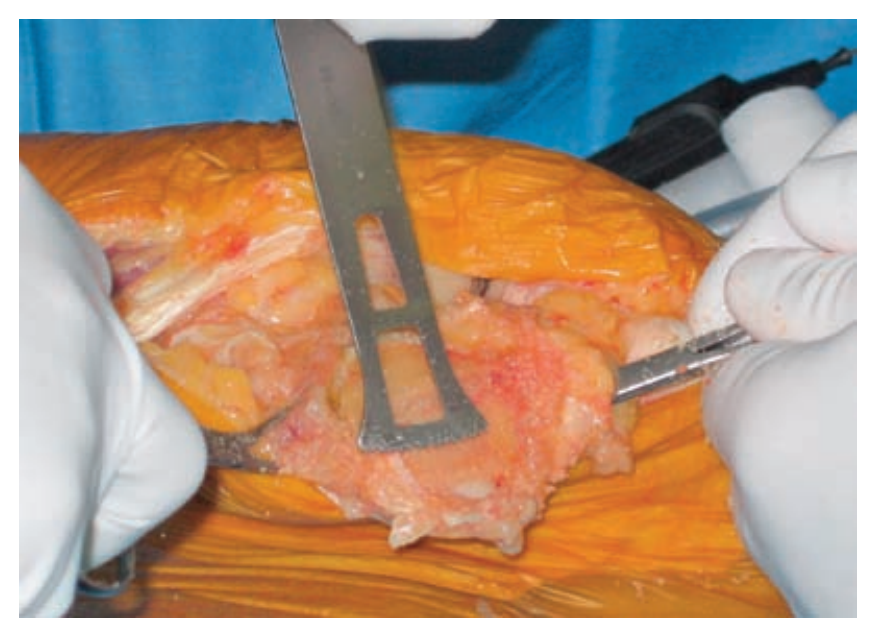

Abb. 2 Dachziegelartige Patellaresektionsarthroplastik.

\section{Abb. 3}

Bestimmung der Größe der femoralen Komponente und Festlegung der Rotation.
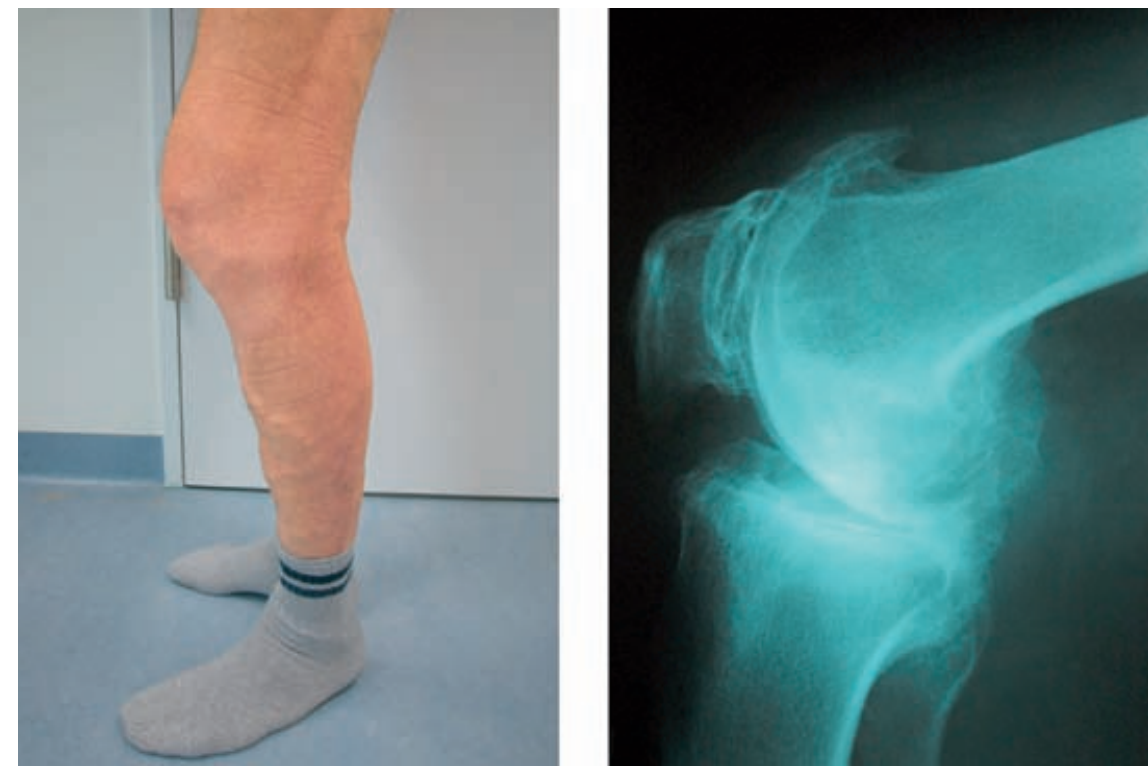

Abb.4 Massive dorsale Osteophyten, die zu einem ausgeprägten klinischen Streckdefizit geführt haben.

arthrose also lateral) als Referenz zu verwenden. Bei minimaler Aufbaustärke des Tibiaplateaus mit Inlay von $8 \mathrm{~mm}$ bedeutet dies eine Resektion von $8 \mathrm{~mm}$. Diese Vorgehensweise ist besonders bei starken Achsdeformitäten und tiefen Defekten angezeigt, um die Resektionshöhe möglichst gering zu halten. Dennoch ist die Gegenseite immer nachzumessen, auch um einen Überblick über das Ausmaß des Defektes zu erhalten (Abb.5). Es ist eher ein „undercutting“ mit einem notwendigen Aufbau zu akzeptieren, als zu viel Knochen an der Tibia zu entfernen, da sich dies natürlich auch auf die Gelenklinie auswirkt. Sofern die Tibia geschnitten ist und Osteophyten entfernt wurden, können nun die Probeimplantate eingebracht und das Ergebnis überprüft werden. Bei persistierenden Instabilitäten ist nun das Weichteilbalancing durchzuführen. Sofern die Stabilität des
Kunstgelenks über den gesamten Bewegungsumfang gegeben ist, kann die definitive Implantation vorgenommen werden.

\section{Ergebnisse konventionelle Endopro- thetik}

Die aktuelle Literatur verfügt über eine Vielzahl von Studien, die die Überlebensrate von Knietotalendoprothesen beleuchtet. Aus der jüngeren Zeit ist die Studie von Himanen [23] hervorzuheben, die multizentrisch 8467 KTP untersucht und eine Überlebensrate von 97\% nach 5 Jahren und 94\% nach 10 Jahren nachweisen kann. Zwei aktuelle „single surgeon“-Studien zeigen Überlebensraten von $92,6 \%$ nach 15 Jahren beim Arthrotiker [12] und 100\% nach 15 Jahren respektive 93,7\% nach 20 Jahren beim Rheumatiker [8].
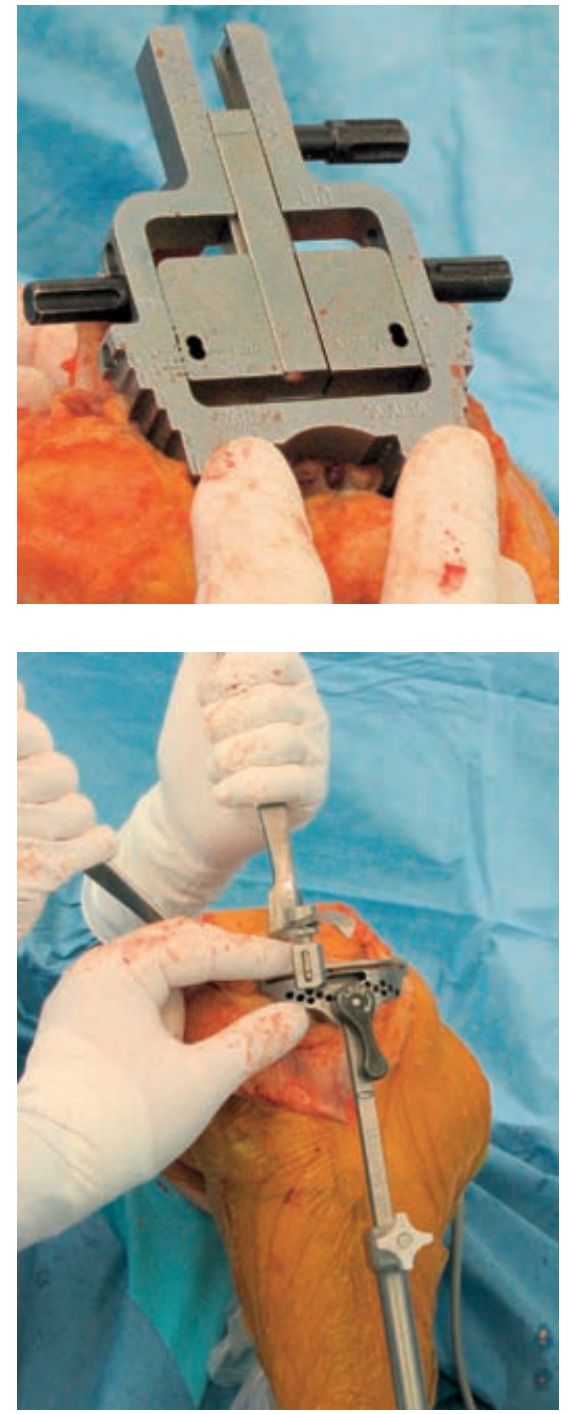

Abb.5 Tibiale Ausrichtung und Ausmessen des tibialen Defekts mit der Messlehre.

Standzeiten von 10 Jahren sind in 95\% der Fälle realistisch.

Oft wird die Frage nach erhöhter Lockerungs- und Komplikationsrate bei Diabetikern und adipösen Patienten besprochen: $\mathrm{Zu}$ dieser Thematik liegen zwei Arbeiten vor:

Meding (2003) findet für Patienten, die an Diabtes mellitus Typ II leiden, eine höhere Revisionsrate: 3,6\% vs. 0,4\% bei gesunden Patienten. Auch scheint das Risiko einer tiefen Infektion in 1,2\% mit D. m. und 0,7\% ohne D.m. höher zu liegen. Stark adipöse Patienten werden von Hamoui (21) untersucht, er findet im Vergleich zum schlanken Kontrollkollektiv zumindest keine radiologischen und klinisch-funktionellen Unterschiede. 


\section{Aktuelle Entwicklungen}

Während bis zum Ende der 90er-Jahre vor allem designspezifische Unterschiede im Mittelpunkt wissenschaftlicher Untersuchungen standen, werden in den letzten Jahren zunehmend die Einflussfaktoren des operativen Eingriffs und der Implantationstechnik als Kriterien für das klinische und funktionelle Ergebnis und den Langzeiterfolg in den Vordergrund der wissenschaftlichen Diskussion gestellt [25, 38, 48]. Der korrekten Implantation der Komponenten und einer damit einhergehenden Rekonstruktion einer neutralen Beinachse wird eine entscheidende Bedeutung für eine optimale Standzeit zugesprochen. In verschiedenen klinischen Verlaufsbeobachtungen konnten erhöhte Lockerungsraten bei Achsabweichungen von mehr als $3^{\circ}$ von der neutralen Beinachse ermittelt werden [13, 19, 27, 43, 45, 51]. Diese Ergebnisse lassen sich weiterhin durch
In-vitro-Untersuchungen im Kniesimulator untermauern, wobei ein deutlich erhöhter Polyethylenverschleiß bereits bei Achsabweichungen von drei Grad und mehr zu finden sind (33).

Studien, die die Präzision der konventionellen Implantationstechnik untersuchten, konnten einen Anteil von bis zu 30\% der Implantationen mit Achsabweichungen von mehr als $3^{\circ}$ im Varus oder Valgus ermitteln $(24,27)$

Tab. 1 Übersicht der Studien zum Vergleich der postoperativen Beinachse navigiert vs. konventionelle Operationstechnik, modifiziert nach Bäthis [2]

\begin{tabular}{|c|c|c|c|c|c|}
\hline Arbeitsgruppe & Studiendesign / & Fallzahl (n) & Beinachse inne & alb $+\mid-3^{\circ}$ & \\
\hline $\begin{array}{l}\text { Mielke et al. } 2001 \\
\text { Z Orthop (37) }\end{array}$ & $\begin{array}{l}\text { Prospektiv, vergleichend } \\
\text { OrthoPilot }^{\circledR}\end{array}$ & 60 & $\begin{array}{l}20 / 30 \\
66 \%\end{array}$ & $\begin{array}{l}24 / 30 \\
80 \%\end{array}$ & $\varnothing$ \\
\hline $\begin{array}{l}\text { Jenny et al. } 2001 \\
\text { Rev Chir Orthop Rep App Mot (28) }\end{array}$ & $\begin{array}{l}\text { Matched Pair } \\
\text { OrthoPilot }{ }^{\circledR}\end{array}$ & 100 & $\begin{array}{l}39 / 50 \\
78 \%\end{array}$ & $\begin{array}{l}47 / 50 \\
94 \%\end{array}$ & $\mathrm{p}<0,05$ \\
\hline $\begin{array}{l}\text { Saragagli a. D. } 2001 \\
\text { Rev Chir Orth Rep App Mot (47) }\end{array}$ & $\begin{array}{l}\text { Prospektiv, randomisiert } \\
\text { OrthoPilot }{ }^{\circledR}\end{array}$ & 50 & $\begin{array}{l}19 / 25 \\
76 \%\end{array}$ & $\begin{array}{l}21 / 25 \\
84 \%\end{array}$ & $\varnothing$ \\
\hline $\begin{array}{l}\text { Sparmann et al. } 2003 \\
\text { J Bone Joint Surg (49) }\end{array}$ & $\begin{array}{l}\text { Prospektiv, randomisiert } \\
\text { Stryker }^{\circledR}\end{array}$ & 240 & $\begin{array}{l}107 / 120 \\
89 \%\end{array}$ & $\begin{array}{l}120 / 120 \\
100 \%\end{array}$ & $p<0,0001$ \\
\hline $\begin{array}{l}\text { Bäthis et al. } 2004 \\
\text { J Bone Joint Surg Br [3] }\end{array}$ & $\begin{array}{l}\text { Prospektiv, randomisiert } \\
\text { BrainLAB }{ }^{\circledR}\end{array}$ & 160 & $\begin{array}{l}62 / 80 \\
78 \%\end{array}$ & $\begin{array}{l}78 / 80 \\
98 \%\end{array}$ & $p<0,001$ \\
\hline $\begin{array}{l}\text { Chauhan et al. } 2004 \\
\text { J Bone Joint Surg Br [5] }\end{array}$ & $\begin{array}{l}\text { Prospektiv, randomisiert } \\
\text { Stryker }^{\circledR}\end{array}$ & 70 & $\begin{array}{l}25 / 35 \\
71 \%\end{array}$ & $\begin{array}{l}30 / 35 \\
86 \%\end{array}$ & $\mathrm{p}<0,032$ \\
\hline $\begin{array}{l}\text { Perlick et al. } 2004 \\
\text { Acta Orthop Scand (40) }\end{array}$ & $\begin{array}{l}\text { Prospektiv, vergleichend } \\
\text { BrainLAB }{ }^{\circledR} \text { (CT-based) }\end{array}$ & 100 & $\begin{array}{l}36 / 50 \\
72 \%\end{array}$ & $\begin{array}{l}46 / 50 \\
92 \%\end{array}$ & $\mathrm{p}<0,01$ \\
\hline $\begin{array}{l}\text { Matsumoto } 2004 \\
\text { Int Orthop (36) }\end{array}$ & matched pair Brain $L A B^{\circledR}$ & 60 & $\begin{array}{l}20 / 30 \\
67 \%\end{array}$ & $\begin{array}{l}28 / 30 \\
93 \%\end{array}$ & $\mathrm{p}<0,05$ \\
\hline $\begin{array}{l}\text { Daubresse } 2005 \\
\text { Acta Orthop Belg [10] }\end{array}$ & $\begin{array}{l}\text { Prospektiv, vergleichend } \\
\text { k.A. }\end{array}$ & 100 & $\begin{array}{l}34 / 50 \\
68 \%\end{array}$ & $\begin{array}{l}50 / 50 \\
100 \%\end{array}$ & $\mathrm{p}<0,001$ \\
\hline $\begin{array}{l}\text { Kim, } 2005 \\
\text { J Arthroplasty (31) }\end{array}$ & $\begin{array}{l}\text { Prospektiv, vergleichend } \\
\text { Stryker }^{\circledR}\end{array}$ & N68/K79 & $\begin{array}{l}58 / 79 \\
73 \%\end{array}$ & $\begin{array}{l}64 / 68 \\
93 \%\end{array}$ & $\mathrm{p}<0,001$ \\
\hline $\begin{array}{l}\text { Chin P, } 2005 \\
\text { J Arthroplasty [7] }\end{array}$ & $\begin{array}{l}\text { Prospektiv, randomisiert } \\
\text { Brain } L A B^{\circledR}\end{array}$ & 60 & $\begin{array}{l}22 / 30 \\
73 \%\end{array}$ & $\begin{array}{l}28 / 30 \\
93 \%\end{array}$ & $\mathrm{p}<0,01$ \\
\hline $\begin{array}{l}\text { Jenny et al. } 2005 \\
\text { J Arthroplasty (28) }\end{array}$ & $\begin{array}{l}\text { Multicenter, gepaart } \\
\text { OrthoPilot Aesculap }{ }^{\circledR}\end{array}$ & 470 & $\begin{array}{l}170 / 235 \\
72 \%\end{array}$ & $\begin{array}{l}217 / 235 \\
92 \%\end{array}$ & $p<0,001$ \\
\hline $\begin{array}{l}\text { Anderson, K.C } 2005 \\
\text { J Arthroplasty [1] }\end{array}$ & $\begin{array}{l}\text { Prospektiv, vergleichend } \\
\text { Stryker }^{\circledR}\end{array}$ & N116/K51 & $\begin{array}{l}42 / 51 \\
84 \%\end{array}$ & $\begin{array}{l}110 / 116 \\
95 \%\end{array}$ & $\mathrm{p}<0,02$ \\
\hline Gesamt & & 1784 & $\begin{array}{l}654 / 865 \\
75,6 \%\end{array}$ & $\begin{array}{l}863 / 919 \\
93,9 \%\end{array}$ & $\begin{array}{l}\chi^{2}-\text { Test } \\
\mathrm{p}<0,0001\end{array}$ \\
\hline $\begin{array}{l}\text { Hart et al. } 2003 \\
\text { Int Orthop (22) }\end{array}$ & $\begin{array}{l}\text { Prospektiv, randomisiert } \\
\text { OrthoPilot Aesculap }{ }^{\circledR}\end{array}$ & 120 & $\begin{array}{l}42 / 60 \\
70 \%\left(+-2^{\circ}\right)\end{array}$ & $\begin{array}{l}53 / 60 \\
88 \%\left(+-2^{\circ}\right)\end{array}$ & $\mathrm{p}<0,02$ \\
\hline $\begin{array}{l}\text { Stöckl, B } 2004 \\
\text { CORR (50) }\end{array}$ & $\begin{array}{l}\text { Prospektiv, randomisiert } \\
\text { Stryker Leibinger }\end{array}$ & 64 & $\begin{array}{l}30 / 32 \\
94 \%\left(+-5^{\circ}\right)\end{array}$ & $\begin{array}{l}32 / 32 \\
100 \%\left(+-5^{\circ}\right)\end{array}$ & $\varnothing$ \\
\hline $\begin{array}{l}\text { Decking, R } 2005 \\
\text { J Arthroplasty [11] }\end{array}$ & $\begin{array}{l}\text { Prospektiv, randomisiert } \\
\text { OrthoPilot Aesculap }\end{array}$ & 52 & $\begin{array}{l}9 / 25 \\
36 \%\left(+-2^{\circ}\right)\end{array}$ & $\begin{array}{l}14 / 27 \\
51 \%\left(+-2^{\circ}\right)\end{array}$ & $p<0,02$ \\
\hline $\begin{array}{l}\text { Bolognesi, M } 2005 \\
\text { CORR [4] }\end{array}$ & $\begin{array}{l}\text { Orthosoft }{ }^{\circledR} \\
\text { comparative retrospective } \\
\text { Component position }\end{array}$ & 100 & $\begin{array}{l}\text { Femoral } \\
\mathrm{N}: 49 / 50(98 \%) \\
\mathrm{K}: 45 / 50(90 \%)\end{array}$ & $\begin{array}{l}\text { Tibial } \\
\text { N: 50/50(100\%) } \\
\text { K: } 46 / 50(92 \%)\end{array}$ & \\
\hline Gesamt & & 2217 & 1081 & 1136 & \\
\hline
\end{tabular}


Diese Tatsache führte dazu, dass sich Navigationssysteme auch für den endoprothetischen Ersatz des Kniegelenks etablierten. In der klinischen Anwendung der Knieendoprothetik hat sich der Vorteil der bildfreien Systeme bezüglich einer direkten intraoperativen Anwendbarkeit ohne vorherige Bildgebung in der Praxis durchgesetzt. Die derzeitig verfügbaren Systeme der bildfreien Navigation erlauben mittlerweile neben der Planung, Darstellung und Kontrolle der notwendigen intraoperativen Knochenresektionen auch eine intraoperative Darstellung der Beinachse, Überprüfungsmöglichkeiten der Kollateralbandstabilität und zum Teil optimierter Möglichkeiten der Implantatausrichtung der Knieprothese $[3,32]$.

Die Navigation ist der Freihandtechnik hinsichtlich der Genauigkeit der Achsrekonstruktion überlegen.

$\mathrm{Zu}$ diesem Themenkomplex liegt eine aktuelle Metaanalyse aus der Arbeitsgruppe der Autoren vor, die eindeutig die Vorteile der Navigation hinsichtlich der Rekonstruktion einer geraden und neutralen Beinachse belegt. Tabelle 1 zeigt die untersuchten Studien [2]. Bislang liegt noch keine Studie vor, die den klinischen Nutzen beweisen kann, da der Endpunkt für diese Studien, die aseptische Lockerung, erst in frühestens 10 Jahren nach Implantation zu erwarten ist.

\section{Schlussfolgerung}

Obwohl sich die Implantation von Knietotalendoprothesen $\mathrm{zu}$ einer Routineoperation an vielen Kliniken etabliert hat, bietet die Operationstechnik einige Fehlermöglichkeiten. Knochenschnittabweichungen, suboptimales Weichteilmanagement und Achsfehler können zu verfrühten Lockerungen führen. Wie die aktuelle Literatur zeigt, ist die Navigation in der Lage, selbst den erfahrenen Operateur bei allen diesen Punkten zu unterstützen. Speziell bei schweren Achsdeformitäten sollte die Unterstützung durch die Navigation bedacht werden.

\section{Literatur}

1 Anderson KC, Buehler KC, Markel DC. Computer assisted navigation in total knee arthroplasty: comparison with conventional methods. J Arthroplasty 2005; 20:132-138

2 Bäthis H, Shafizadeh S, Paffrath T, Simanski S, Grifka J, Lüring C. Sind navigierte Knieendoprothesen tatsächlich präziser implantiert? Eine Metaanalyse vergleichender Stu- dien - In press: Der Orthopäde 2006; 35: 1056-1065

3 Bäthis H, Perlick L, Tingart M, Luring C, Zurakowski D, Grifka J. Alignment in total knee arthroplasty. A comparison of computer-assisted surgery with the conventional technique. J Bone Joint Surg Br 2004; 86:682687

${ }^{4}$ Bolognesi M, Hofmann A. Computer navigation versus standard instrumentation for TKA: a single-surgeon experience. Clin Orthop Relat Res 2005; 440:162-169

5 Chauhan SK, Scott RG, Breidahl W, Beaver RJ. Computer-assisted knee arthroplasty versus a conventional jig-based technique. A randomised, prospective trial. J Bone Joint Surg Br 2004; 86:372-377

6 Chen FS, Scher DM, Clancy RM, Ayesha V-Y, DiCesare PE. In vitro and in vivo activation of polymorphonuclear leukocytes in response to particulate debris. J Biomed Mater Res 1999; 48:904-912

7 Chin PL, Yang KY, Yeo SJ, Lo NN. Randomized control trial comparing radiographic total knee arthroplasty implant placement using computer navigation versus conventional technique. J Arthroplasty 2005; 20:618-626

8 Crowder AR, Duffy GP, Trousdale RT. Longterm results of total knee arthroplasty in young patients with rheumatoid arthritis. J Arthroplasty 2005; 20[7]: 12-16

${ }^{9}$ Dalury DF, Jiranek WA. A comparison of the midvastus and paramedian approaches for total knee arthroplasty. J Arthroplasty 1999; 14:33-37

10 Daubresse F, Vajeu C, Loquet J. Total knee arthroplasty with conventional or navigated technique: comparison of the learning curves in a community hospital. Acta Orthop Belg 2005; 71:710-713

11 Decking R, Markmann Y, Fuchs J, Puhl W, Scharf HP. Leg axis after computer-navigated total knee arthroplasty: a prospective randomized trial comparing computer-navigated and manual implantation. J Arthroplasty 2005; 20:282-288

12 Dixon MC, Brown RR, Parsch D, Scott RD. Modular fixed-bearing total knee arthroplasty with retention of the posterior cruciate ligament. A study of patients followed a minimum of fifteen years. JBJS Am. 2005; 87[2]: 598-603

13 Elloy MA, Manning MP, Johnson R. Accuracy of intramedullary alignment in total knee replacement. J.Biomed.Eng 1992; 14:363370

14 Engh GA. The difficult knee. Severe varus and valgus. Clin Orthop 2003; 416: 58-63

15 Engh GA, Parks NL. Surgical technique of the midvastus arthrotomy. Clin Orthop 1998; 331: 270-284

16 Erkes F. Weitere Erfahrungen mit physiologischer Schnittführung zur Eröffnung des Kniegelenks. Bruns Beit Klein Chir 1929; 147: 221-232

17 Faure BT, Benjamin JB, Lindsey JB, Volz RG Schutte D. Comparison of the subvastus and paramedian surgical approaches in bilateral knee arthroplasty. J Arthroplasty 1993; 8: 511-516

18 Freeman MA, Todd RC, Bamert P, Day WH ICLH arthroplasty of the knee 1968-1977. J Bone Joint Surg Br 1978; 60: 339-344

19 Freund DA, Dittus RS, Fitzgerald J, Heck D. Assessing and improving outcomes: total knee replacement. Health Serv Res 1990; 25:723-726

20 Glant TT, Jacobs JJ. Response of three murine macrophage populations to particulate debris: bone resorption in organ cultures. J Orthop Res 1994; 12:720-731
21 Hamoui N, Kantor S, Vince K, Crookes PF. Long-term outcome of total knee replacement: does obesity matter? Obes Surg 2006; 16[1]: 35-38

22 Hart R, Janecek M, Chaker A, Bucek P. Total knee arthroplasty implanted with and without kinematic navigation. Int Orthop 2003; 27:366-369

23 Himanen AK, Belt E, Nevalainen J, Hamalainen M, Lehto MU. Survival of the AGC total knee arthroplasty is similar for arthrosis and rheumatoid arthritis. Finnish Arthroplasty Register report on 8467 operations carried out between 1985 and 1999. Acta Orthop Scand 2005; 76[1] 85-8

24 Hood RW, Vanni M, Insall JN. The correction of knee alignment in 225 consecutive total condylar knee replacements. Clin.Orthop. 1981: 94-105

25 Insall JN, Easley ME. Surgical techniques and instrumentation in total knee arthroplasty. Churchill Livingston, Livingstone Philadelphia 2001; 3rd Edition: 1553-1620

26 Jacobs JJ, Gilbert JL, Urban RM. Corrosion of metal orthopaedic implants. J Bone Joint Surg 1998; 80A:268-282

27 Jeffery RS, Morris RW, Denham RA. Coronal alignment after total knee replacement. J. Bone Joint Surg.Br. 1991; 73:709-714

28 Jenny JY, Boeri C. [Computer-assisted implantation of a total knee arthroplasty: a case- controlled study in comparison with classical instrumentation]. Rev.Chir Orthop. Reparatrice Appar.Mot. 2001; 87:645-652

29 Jenny JY, Clemens U, Kohler S, Kiefer H, Konermann W, Miehlke RK. Consistency of implantation of a total knee arthroplasty with a non-image-based navigation system: a casecontrol study of 235 cases compared with 235 conventionally implanted prostheses [In Process Citation]. J Arthroplasty 2005; 20:832-889

30 Kanamiya T, Whiteside LA, Nakamura T, Mihalko WM, Steiger J, Naito M. Effect of selctive lateral ligament release on stability in knee arthroplasty. Clin Orthop 2002; 404: 24-27

31 Kim SJ, MacDonald M, Hernandez J, Wixson RL. Computer assisted navigation in total knee arthroplasty: improved coronal alignment. J Arthroplasty 2005; 20:123-131

32 Kinzl L, Gebhard F, Keppler P. [Total knee arthroplasty--navigation as the standard]. Chirurg 2004; 75:976-981

33 Loer I, Plitz W. [Tibial malalignment of mobile-bearing prostheses--a simulator study]. Orthopade 2003; 32:296-304

34 Maestro A, Suarez MA, Rodriguez L, Guerra $C$, Murcia A. The midvastus surgical approach in total knee arthroplasty. Int Orthop 2000; 24: 104-107

35 Maric Z. The standard vs. the subvastus approach for total knee arthroplasty. Orthop Trans 1991; 15: 43-47

36 Matsumoto T, Tsumura N, Kurosaka M, Muratsu H, Kuroda R, Ishimoto K, et al. Prosthetic alignment and sizing in computer-assisted total knee arthroplasty. Int Orthop 2004; 28:282-285

37 Mielke RK, Clemens U, Jens JH, Kershally S. Navigation in der Knieendoprothetik - vorläufige klinische Erfahrungen und prospektiv vergleichende Studie gegenüber konventioneller Implantationstechnik. Z.Orthop. Ihre Grenzgeb. 2001; 139:109-116

38 Olcott CW, Scott RD. A comparison of 4 intraoperative methods to determine femoral component rotation during total knee arthroplasty. J.Arthroplasty 2000; 15:22-26

39 Otani T, Whiteside LA, White SE. Cutting errors in preparation of femoral components 
in total knee arthroplasty. J Arthroplasty 1993; 8: 503-510

40 Perlick L, Bäthis H, Tingart M, Perlick C, Grifka J. Navigation in total-knee arthroplasty: CT-based implantation compared with the conventional technique. Acta Orthop Scand 2004; 75:464-470

41 Peters PC, Knezevich S, Engh GA, Preidis FE, Dwyer KA. Comparison of subvastus quadrizeps-sparing and standard quadrizeps-splitting approaches in total and unicompartmental knee arthroplasty. Orthop Trans 1992; 16: 615-619

42 Plaskos C, Hodgson AJ, Inkpen K, McGraw RW. Bone cutting errors in total knee arthroplasty. J Arthroplasty 2002; 17:698-705

43 Rand JA, Coventry MB. Ten-year evaluation of geometric total knee arthroplasty. Clin.Orthop. 1988; 168-173

${ }^{44}$ Reed SC, Gollish J. The accuracy of femoral intramedullary guides in total knee arthroplasty. J Arthroplasty 1997; 12[6]: 677-682

45 Ritter MA, Faris PM, Keating EM, Meding JB 1994; Postoperative alignment of total knee replacement. Its effect on survival. Clin Orthop 153-156

46 Robertsson O, Dunbar M, Pehrsson T, Knutson K, Lidgren L. Patient satisfaction after knee arthroplasty: a report on 27,372 knees operated on between 1981 and 1995 in Sweden. Acta Orthop Scand 2000; 71: 262-267

47 Saragaglia D, Picard F, Chaussard C, Montbarbon E, Leitner F, Cinquin P. Computer-assisted knee arthroplasty: comparison with a conventional procedure. Results of 50 cases in a prospective randomized study. Rev.Chir Orthop.Reparatrice Appar.Mot. 2001; 87:1828

48 Scuderi GR, Scott WN. Total knee arthroplasty. What have we learned? Am.J.Knee.Surg. 1996; 9:73-75

${ }^{49}$ Sparmann M, Wolke B, Czupalla H, Banzer D, Zink A. Positioning of total knee arthroplasty with and without navigation support. A prospective, randomised study. J Bone Joint Surg Br 2003; 85:830-835

50 Stöckl B, Nogler M, Rosiek R, Fischer M, Krismer M, Kessler O. Navigation improves accuracy of rotational alignment in total knee arthroplasty. Clin Orthop Relat Res 2004; 180 186

51 Tew M, Waugh W. Tibiofemoral alignment and the results of knee replacement. J.Bone Joint Surg.Br. 1985; 67:551-556
Priv.-Doz. Dr. med. Christian Luring zert. Gesundheitsökonom/Assistenzarzt Prof. Dr. med. Joachim Grifka

Direktor

Orthopädische Klinik, Universität Regensburg Asklepios Klinikum Bad Abbach

Kaiser-Karl-V.-Allee 3

93077 Regensburg

\section{Holger Bäthis}

Oberarzt

Klinik für Unfallchirurgie und Orthopädie

Städt. Klinikum Köln-Merheim Lehrstuhl der Universität

Witten-Herdecke

Ostmerheimer Straße 200 51109 Köln 